\title{
Vortex Blooming
}

Weiyu Shen ${ }^{1}$, Jie Yao², Fazle Hussain², Yue Yang' ${ }^{1}$ College of Engineering, Peking University ${ }^{2}$ Department of Mechanical Engineering, Texas Tech University

As basic topological structures, vortex torus unknots, knots, and links play essential roles in various fluid systems, such as hydrodynamic flows and plasmas. Their different topological evolutions, which depend on initial geometry, can significantly affect flow dynamics and turbulence cascade.

The evolutions of coiled vortex loops spirally wound on a torus with different winding numbers are visualized at the vortex Reynolds number $R e$ (E circulation/viscosity) $=2000$. Vorticity isosurfaces are color-coded by the

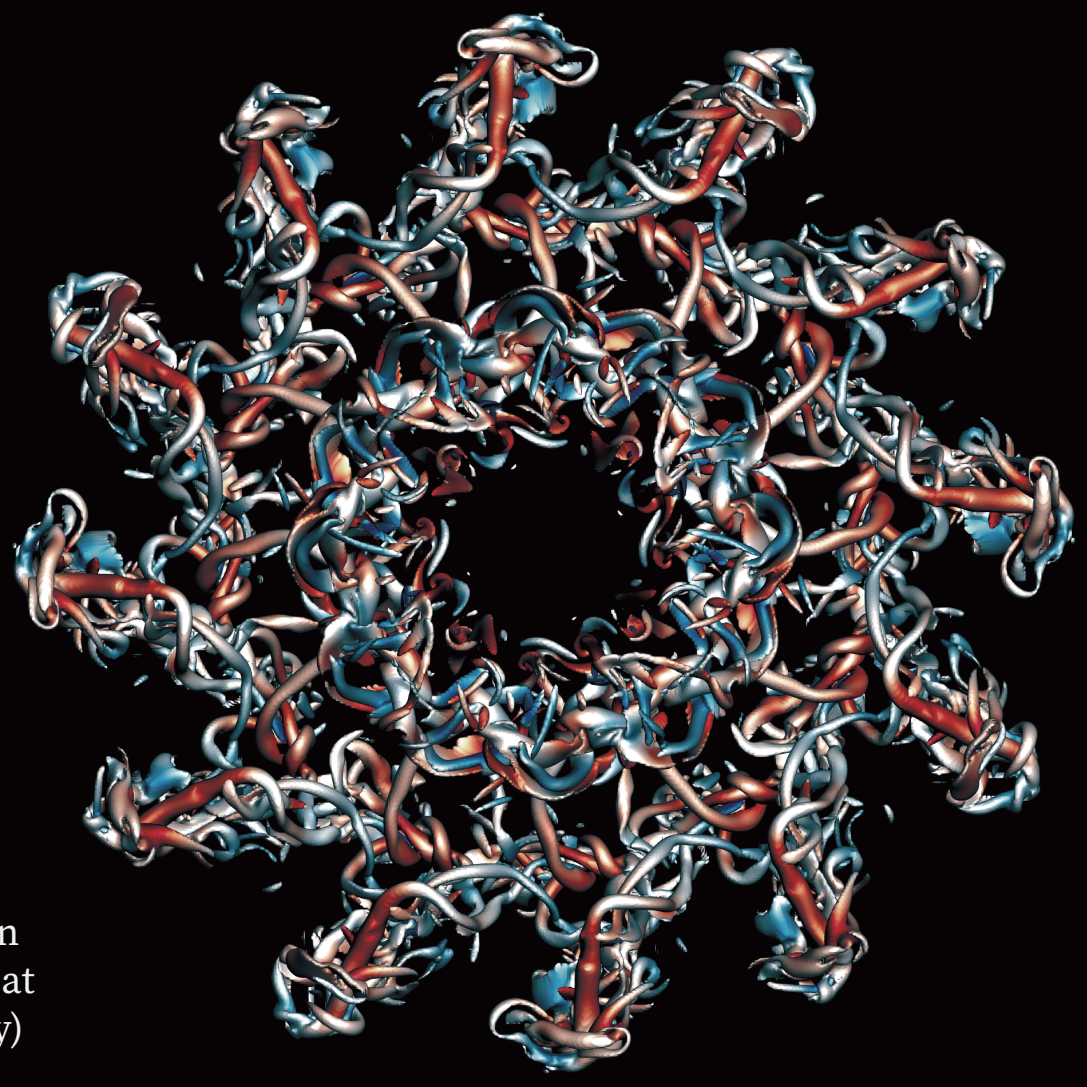
(positive and negative) helicity density.
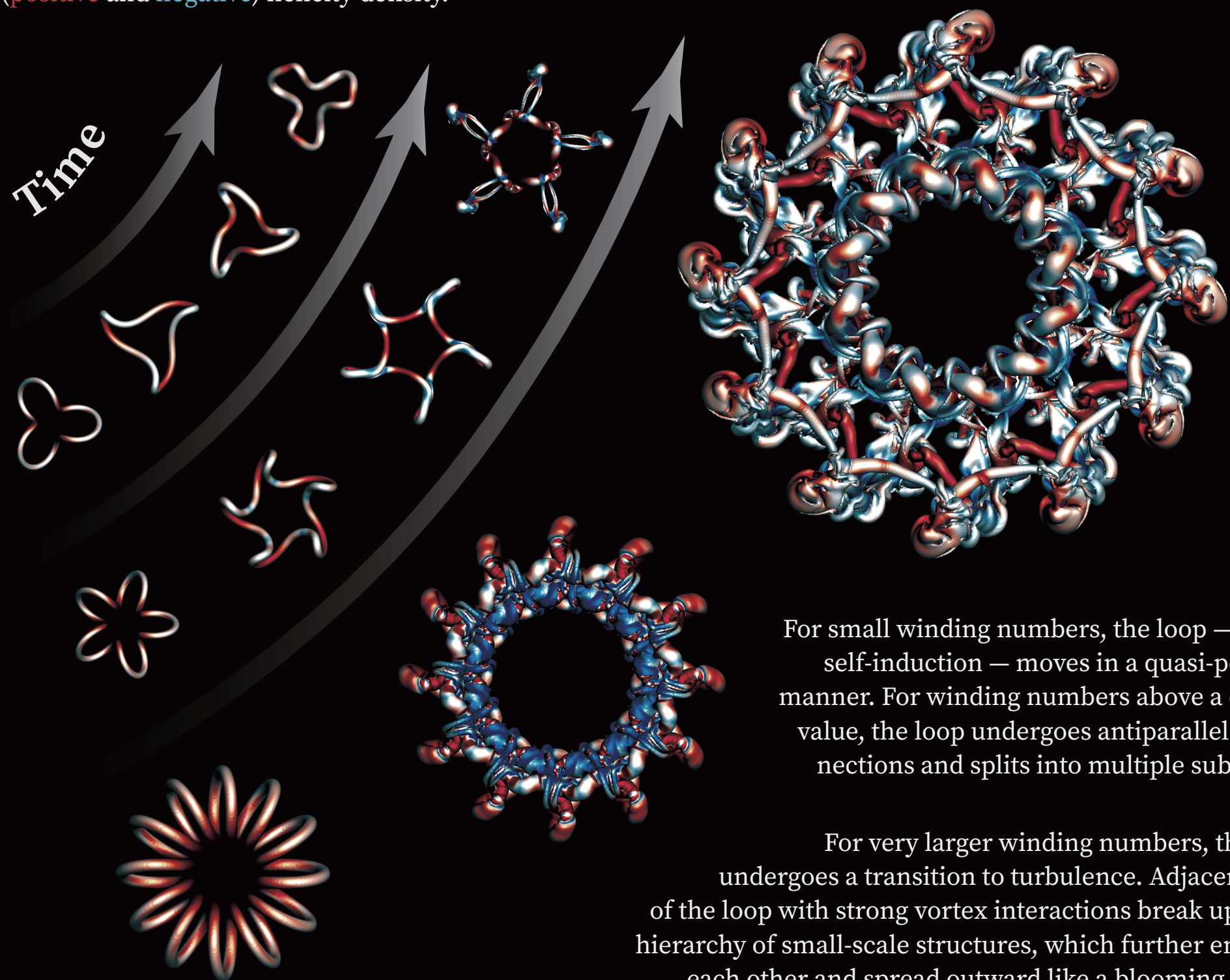
For small winding numbers, the loop - under self-induction - moves in a quasi-periodic manner. For winding numbers above a critical value, the loop undergoes antiparallel recon- nections and splits into multiple sub-loops.

For very larger winding numbers, the loop undergoes a transition to turbulence. Adjacent coils of the loop with strong vortex interactions break up into a hierarchy of small-scale structures, which further entangle each other and spread outward like a blooming flower. 